\title{
On Contractive Semigroups and Uniform Asymptotic Stability
}

\author{
Philip R. Meyers* \\ Institute for Basic Standards, National Bureau of Standards, \\ Washington, D. C. 20234
}

(March 31, 1970)

\begin{abstract}
This paper calls attention to the equivalence between two well-known mathematical ideas: contraction mappings (in the sense of Banach) and asymptotic stability. The equivalence is formalized by defining a flow (representing the possible movements over time of some system through its state space) as a continuous one-parameter semigroup of operators on a metric space, and then showing that these operators are all contractions (in suitably revised metrics) if and only if there is a uniformly asymptotically stable equilibrium point. Generalizations to other operator semigroups are also given.
\end{abstract}

Key words: Contractions; control theory; functional analysis; semigroup; stability theory; topology.

\section{Introduction}

Our aim is to point out, and to make explicit, the relationship between two well-known mathematical ideas: contraction mappings (in the sense of Banach) and asymptotic stability. In this preliminary section we collect the relevant definitions and background material, and state our principal results. The proofs of these results are given in the following section, and various generalizations are given in the concluding part.

Let $(X, d)$ be a metric space, and recall that a map $f: X \rightarrow X$ is called a contraction of $(X, d)$ if there exists $\lambda \epsilon(0,1)$, known as a contraction constant for $f$, such that

$$
d(f(x), f(y)) \leqslant \lambda d(x, y)
$$

for all $x, y \in X$. (Such an $f$ is necessarily continuous.) For present purposes, the Banach Contraction Principle can be conveniently stated as follows:

THEOREM A: Let $\mathrm{f}$ be a contraction of a complete ${ }^{1}$ metric space $(\mathrm{X}, \mathrm{d})$. Then there exists $\xi \epsilon \mathrm{X}$ such that

(i) $f(\xi)=\xi$,

(ii) $f^{n}(x) \rightarrow \xi \quad$ for each $x \in \mathrm{X}$,

(jii) $f^{n}(U) \rightarrow\{\xi\} \quad$ for some neighborhood $\mathrm{U}$ of $\xi$.

It is not difficult to see a connection between conditions (i), (ii), and (iii), and the definition of asymptotic stability. This connection is formalized below. The main tool is the following converse to Theorem A, proved by the author in [1]: ${ }^{2}$

THEOREM B: Let $\mathrm{f}$ be a continuous self-mapping of a metrizable topological space $\mathrm{X}$, which for some $\xi \epsilon \mathrm{X}$ satisfies the three conditions of Theorem A. Then for each $\lambda \epsilon(0,1)$ there exists a metric

*Present address: IBM World Trade Corporation, 821 United Nations Plaza, New York, New York 10017.

1 The completeness hypothesis is inessential, but its omission would require more complicated statements involving equivalent Cauchy sequences.

${ }^{2}$ Figures in brackets indicate the literature references at the end of this paper. 
$\mathrm{d}_{\lambda}$, which is compatible with the topology of $\mathrm{X}$ and can be chosen complete if $\mathrm{X}$ admits a complete metric, such that $\mathrm{f}$ is a contraction of $\left(\mathrm{X}, \mathrm{d}_{\lambda}\right)$ with contraction constant $\lambda$.

Now let $(X, d)$ be a metric space, and $S$ a commutative topological semigroup (written additively) with zero element. Then a family $\left\{T_{s}: s \epsilon S\right\}$ of continuous self-mappings of $X$ (not necessarily homeomorphisms) will be called an $S$-semigroup of operators on $X$ if it obeys

the semigroup condition

$$
T_{o}(x)=x \quad x \in X,
$$

$$
T_{s}\left(T_{t}(x)\right)=T_{s+t}(x) \quad s, t \in S ; x \in X,
$$

and the continuity condition that for each $t \epsilon S$,

$$
\sup \left\{d\left(T_{t}(x), T_{s}(x)\right): x \in X\right\} \rightarrow 0 \quad \text { as } s \rightarrow t .
$$

The semigroup of operators will be called contractive if for some $\lambda \epsilon(0,1)$ there is a family $\left\{d_{s}: s \epsilon S\right\}$ of metrics of $X$, each giving the same topology as that of $(X, d)$, such that $T_{s}$ is a contraction of $\left(X, d_{s}\right)$ with contraction constant $\lambda$ for each $s \epsilon S-\{0\}$. (It is natural to ask whether $d_{s}$ can be chosen independent of $s$. Such questions are addressed in [2] and [3].)

In the motivating situations $X$ is the space of possible "states" of a system whose evolution is "stationary" or "autonomous" (in the differential-equation case, $\dot{x}=f(x)$ rather than $\dot{x}=f(x, t)$ ), while $S$ is a subsemigroup of the additive semigroup $R^{+}$of nonnegative real numbers. Thus we have the interpretation

$$
\begin{gathered}
T_{s}(x)=\text { state of system at time } s \geqslant 0 \\
\text { if } x \text { is its initial state. }
\end{gathered}
$$

Theorem 1: For an $\mathrm{R}^{+}$-semigroup $\left\{\mathrm{T}_{t}: \mathrm{t} \epsilon \mathrm{R}^{+}\right\}$of operators on $\mathrm{X}$ to be contractive, it suffices (and is clearly necessary) that some one $\mathrm{T}_{t}$ obeys the three conditions of Theorem $A$.

Extensions to more general $S$ will be given in the final section. We turn now to the definition of asymptotic stability. In view of the interpretation of $T_{s}(x)$ given above, it is natural to call $\xi \in X$ an equilibrium state if it is a common fixed point of the semigroup of operators, i.e.,

$$
T_{s}(\xi)=\xi \quad \text { for } s \epsilon S .
$$

For this to be true, it suffices that some $T_{t}$ have $\xi$ as unique fixed point, since then for any $s \epsilon S$

$$
T_{s}(\xi)=T_{s}\left(T_{t}(\xi)\right)=T_{s+t}(\xi)=T_{t+s}(\xi)=T_{t}\left(T_{s}(\xi)\right)
$$

so that $T_{s}(\xi)$ is a fixed point of $T_{t}$, implying $T_{s}(\xi)=\xi$ as desired. In this connection note that (i) and (ii) of Theorem A imply that $\xi$ is the only fixed point of $f$.

Recall that an equilibrium state $\xi$ is called stable if for each neighborhood $V$ of $\xi$ there is a neighborhood $W$ of $\xi$ such that

$$
x \in W \text { implies } T_{s}(x) \epsilon V \text { for all } s \epsilon S \text {. }
$$

For the case $S \subseteq R^{+}$, the stable equilibrium state $\xi$ is called asymptotically stable if there is a neighborhood $U$ of $\xi$ such that

$$
x \in U \text { implies } T_{s}(x) \rightarrow \xi \quad \text { as } s \rightarrow \infty,
$$

and is called uniformly asymptotically stable if $U$ can be so chosen that

$$
T_{s}(U) \rightarrow\{\xi\} \quad \text { as } s \rightarrow \infty .
$$


The relation between this concept and that of a contraction mapping is given in the second of our principal results:

THEOREM 2: The equilibrium state $\xi$ of an $\mathrm{R}^{+}$-semigroup of operators is uniformly asymptotically stable if and only if $\xi$ has a neighborhood $\mathrm{X}_{0} \subseteq \mathrm{X}$ such that the restriction of the semigroup to $\mathrm{X}_{0}$ is a contractive semigroup.

In many applications $\xi$ will necessarily have a compact neighborhood (e.g., when $X$ is a finitedimensional Euclidean space). It will be apparent from the proofs that for such cases the continuity condition (1.1) can be weakened to a requirement that, for each compact subset $C$ of $X$ and each $t \epsilon S$,

$$
\sup \left\{d\left(T_{t}(x), T_{s}(x)\right): x \in C\right\} \rightarrow 0 \quad \text { as } s \rightarrow t \text {. }
$$

\section{Proofs of Theorems 1 and 2}

Throughout this section $\left\{T_{s}: s \in R^{+}\right\}$is an $R^{+}$-semigroup of operators on metric space $(X, d)$, and for $x \in X$ and $r>0$ we set

$$
S(x, r)=\{y: y \in X, d(x, y) \leqslant r\} .
$$

The proofs of theorems 1 and 2 both use the following lemma.

LEMmA: Let $\xi \in \mathrm{X}$ be an equilibrium state. For each $\mathrm{t}>0$ and $\eta>0$, there is a $\delta>0$ such that

$$
T_{s}(S(\xi, \delta)) \subseteq S(\xi, \eta) \quad \text { for } 0 \leqslant s \leqslant t
$$

Proof: If not, there is a $t>0$, an $\eta>0$, a positive sequence $\delta_{n} \rightarrow 0$, and associated sequences of points $x_{n} \in S\left(\xi, \delta_{n}\right)$ and of numbers $s(n) \epsilon[0, t]$ such that

$$
T_{s(n)}\left(x_{n}\right) \epsilon X-S(\xi, \eta) .
$$

By passing to a subsequence if necessary, we may suppose that $s(n) \rightarrow s \epsilon[0, t]$. Then

$$
d\left(\xi, T_{s(n)}\left(x_{n}\right)\right) \leqslant d\left(T_{s}(\xi), T_{s}\left(x_{n}\right)\right)+d\left(T_{s}\left(x_{n}\right), T_{s(n)}\left(x_{n}\right)\right) .
$$

The limit as $n \rightarrow \infty$ of the first term on the right vanishes because $x_{n} \rightarrow \xi$, while the continuity of the semigroup of operators ensures the same for the second term. So we have a contradiction, completing the indirect proof of the lemma.

For the proof of Theorem 1, it suffices (by Theorem B) to assume that some $T_{t}$ obeys conditions (i), (ii), and (iii) of Theorem $\mathrm{A}$, and to show that any $T_{s}(s>0)$ does the same. It was shown below the statement of Theorem 1 that $T_{s}$ satisfies (i), with the same fixed point $\xi$ as for $T_{t}$. Note that $t>0$, since $T_{0}$ cannot satisfy (ii) . . . except for the trivial case $X=\{\xi\}$ when the theorem is true anyhow.

Condition (ii) for $T_{s}$ is verified by applying the lemma in the following way: Consider any $x \in X$ and any $\eta>0$. By (ii) for $T_{t}$, we can choose $N>0$ so large that

$$
d\left(T_{t}^{n}(x), \xi\right)<\delta, \quad \text { for } n \geqslant N,
$$

where $\delta$ is related to $t$ and $\eta$ as in the lemma. Next choose $M>0$ so large that

$$
m s \geqslant N t \quad \text { for } m \geqslant M \text {. }
$$

Then for $m \geqslant M$ we have

so that by the lemma

while

$$
m s=n t+\sigma \quad(n \geqslant N, 0 \leqslant \sigma<t),
$$

$$
T_{\sigma}(S(\xi, \delta)) \subseteq S(\xi, \eta)
$$

$$
T_{s}^{m}(x)=T_{s m}(x)=T_{\sigma}\left(T^{n}(x)\right) \epsilon T_{\sigma}(S(\xi, \delta)),
$$


hence for $m \geqslant M=M(\eta), T_{s}^{m}(x) \in S(\xi, \eta)$, proving (ii).

To prove (iii) for $T_{s}$, consider any $\eta>0$, let $\delta$ be related to $t$ and $\eta$ as in the lemma, and (since (iii) holds for $T_{t}$ ) let $U$ be a neighborhood of $\xi$ such that $T_{t}^{n}(U) \rightarrow\{\xi\}$. Choose $N>0$ so large that

$$
T^{n}(U) \subseteq S(\xi, \delta) \quad \text { for } n \geqslant N,
$$

and $M$ so large that $m s>N t$ for $m \geqslant M$. Then for $m \geqslant M$ we can proceed as above to prove $T_{s}^{m}(U) \subseteq S(\xi, \eta)$, completing the proof.

We turn now to the first half of the proof of Theorem 2. Assuming the existence of a neighbor$\operatorname{hood} X_{0}$ of the equilibrium state $\xi$ such that the restriction of the semigroup to $X_{0}$ is contractive, we wish to show that $\xi$ is uniformly asymptotically stable.

Let $V$ be any neighborhood of $\xi$, consider any $t>0$, and choose $\eta>0$ such that

$$
S(\xi, \eta) \subseteq V \cap X_{0}
$$

Then choose $\delta$ related to $t$ and $\eta$ as in the lemma, implying $\delta \leqslant \eta$. There is a metric $d_{t}$ on $X_{0}$, yielding the same topology as $d$, such that $T_{t} \mid X_{0}$ is a contraction on $\left(X_{0}, d_{t}\right)$. Thus there is a $\Delta>0$ such that

$$
W=\left\{y: y \in \mathrm{X}_{0}, d_{t}(y, \xi)<\Delta\right\} \subseteq S(\xi, \delta)
$$

and also such that $T_{t}(W) \subseteq W$. Then for any $s \geqslant 0$, written

$$
s=n t+\sigma \quad(n \geqslant 0,0 \leqslant \sigma<t),
$$

we have

$$
T_{s}(W)=T_{\sigma}\left(T_{t}^{n}(W)\right) \subseteq T_{\sigma}(W) \subseteq T_{\sigma}(S(\xi, \delta)) \subseteq S(\xi, \eta) \subseteq V,
$$

proving that $\xi$ is stable.

Moreover, there is a neighborhood $U$ of $\xi$ (independent of $V$ ) such that $U \subseteq X_{0}, T_{t}(U) \subseteq X_{0}$, and $T_{t}^{n}(U) \rightarrow\{\xi\}$. Given $V$, choose $\eta$ and $\delta$ as above. Pick $N>0$ so large that

$$
T_{t}^{n}(U) \subseteq S(\xi, \delta) \quad \text { for } n \geqslant N
$$

Then any $s \geqslant N t$ can be written

so that

$$
s=n t+\sigma \quad(n \geqslant N, 0 \leqslant \sigma<t)
$$

$$
T_{s}(U)=T_{\sigma}\left(T t^{n}(U)\right) \subseteq T_{\sigma}(S(\xi, \delta)) \subseteq S(\xi, \eta) \subseteq V
$$

Hence $T_{s}(U) \rightarrow\{\xi\}$ as $s \rightarrow \infty$, implying that $\xi$ is uniformly asymptotically stable.

For the converse half of Theorem 2, assume $\xi$ is a uniformly asymptotically stable equilibrium state, and define

$$
X_{0}=\left\{x: x \in X, T_{s}(x) \rightarrow \xi \quad \text { as } s \rightarrow \infty\right\}
$$

The definition of asymptotic stability ensures that $X_{0}$ contains a neighborhood of $\xi$, and so is itself such a neighborhood. The semigroup property implies that $T_{s}\left(X_{0}\right) \subseteq X_{0}$ for each $s \epsilon R^{+}$, so that $\left\{T_{s} \mid X_{0}: s \in R^{+}\right\}$is an $R^{+}$-semigroup of operators on $X_{0}$.

Choose any $t>0$. The definition of $X_{0}$ ensures that $T_{t} \mid X_{0}$ satisfies conditions (i) and (ii) of Theorem A, so only (iii) need be verified. The uniform asymptotic stability of $\xi$ ensures the existence of a neighborhood $U$ (in $X_{0}$, and hence in $X$ ) of $\xi$ such that $T_{s}(U) \rightarrow\{\xi\}$ as $s \rightarrow \infty$, and hence in particular such that $T_{t}^{n}(U)=T_{n t}(U) \rightarrow\{\xi\}$. So (iii) also holds, and an appeal to Theorem B completes the proof of Theorem 2. 


\section{Remarks and Generalizations}

Our first group of remarks pertain to extending Theorem 1 from $S=R^{+}$to other semigroups. We begin with the discrete case, i.e., $S$ is algebraically arbitrary but has the discrete topology (more precisely, the topology of $S$ is irrelevant). The simplest instance is that in which $S$ is the semigroup $I^{+}$of nonnegative integers, a situation arising for example in connection with sampleddata systems. The next theorem generalizes a result, for $S=I^{+}$, proved in [1] as a simple corollary to Theorem B. Its statement uses the fact that the definition of "contractive," introduced earlier only for semigroups of operators on $(X, d)$, is equally applicable to any family of self-mappings of $(X, d)$.

THEOREM 3: The semigroup $\left\{\mathrm{T}_{\mathrm{S}}: \mathrm{s} \epsilon \mathrm{S}\right\}$ of operators is contractive if and only if there is a generating subset $\mathrm{T}$ of $\mathrm{S}$ such that $\left\{\mathrm{T}_{\mathrm{t}}: \mathrm{t} \epsilon \mathrm{T}\right\}$ is contractive.

Proof: The "only if" direction is obvious; just take $T=S$. Suppose now that $\left\{T_{t}: t \epsilon T\right\}$ is contractive, where $T$ generates $S$. Consider any $s \in S-\{0\}$. Since $T$ is a generating set, we have

$$
s=t(1)+t(2)+\ldots+t(m)
$$

where each $t(j) \epsilon T-\{0\}$. Thus, with composition of operators written multiplicatively, we have

$$
T_{s}=T_{t(1)} T_{t(2)} \ldots T_{t(m)}
$$

Each $T_{t(j)}$ has a unique fixed point, and since they commute they have the same fixed point $\xi$. Thus $T_{s}$ has $\xi$ as fixed point, and so satisfies condition (i) of Theorem A.

To each $T_{t(j)}$ there corresponds a neighborhood $U_{j}$ of $\xi$ such that $T_{t(j)}^{n}\left(U_{j}\right) \rightarrow\{\xi\}$. As shown in the proof of Theorem $\mathrm{B}$ in [1], $U_{j}$ can be assumed chosen so that $T_{t(j)}\left(U_{j}\right) \subseteq U_{j}$. Let $U=\bigcap_{1}^{m} U_{j}$ and note that, by commutativity,

$$
T_{s}^{n}=T_{t(1)}^{n} T_{t(2)}^{n} \ldots T_{t(m)}^{n} \text {. }
$$

Also, let $V$ be any neighborhood of $\xi$.

Consider any $x \in X$. Choose $N$ so large that, for $1 \leqslant j \leqslant m$,

$$
\begin{array}{ll}
\text { (a) } T_{t(j)}^{n}\left(U_{j}\right) \subseteq U \cap V \subseteq U_{j}, & \text { for } n \geqslant N, \\
\text { (b) } T_{t(m)}^{n}(x) \epsilon U \cap V \subseteq U_{m-1}, & \text { for } n \geqslant N .
\end{array}
$$

Then for $n \geqslant N$,

$$
T_{s}^{n}(x) \epsilon T_{t(1)}^{n} T_{t(2)}^{n} \quad \ldots T_{t(m-1)}^{n}(U \cap V) \subseteq \ldots \subseteq T_{t(1)}^{n}(U \cap V) \subseteq U \cap V \subseteq V,
$$

proving that $T_{s}^{n}(x) \rightarrow \xi$.

If $N$ is chosen so large that (a) holds for $1 \leqslant j \leqslant m$, then the same inclusions show that $T_{s}^{n}(U) \subseteq V$ for $n \geqslant N$, so that $T_{s}^{n}(U) \rightarrow\{\xi\}$ and an application of Theorem B completes the proof.

For the case $S=I^{+}$, where $T$ can be taken to have a single element, there is clearly an analog of Theorem 2 relating contractiveness to uniform asymptotic stability.

The next case treated is essentially an amalgam of the discrete situation and the case $S=R^{+}$.

THEOREM 4: Let $\mathrm{S}$ be a convex cone, with 0 as apex, in a real topological vector space. Then an $\mathrm{S}$-semigroup $\left\{\mathrm{T}_{\mathrm{s}}: \mathrm{s} \in \mathrm{S}\right\}$ of operators is contractive if and only if there is a subset $\mathrm{T}$ of $\mathrm{S}$ such that $\left\{\mathrm{T}_{\mathrm{t}}: \mathrm{t} \epsilon \mathrm{T}\right\}$ is contractive and each $\mathrm{s} \epsilon \mathrm{S}$ is a nonnegative finite linear combination of members of $\mathrm{T}$.

Proof: As before, the choice $T=S$ proves the "only if". Now suppose there is a subset $T$ with the specified properties. Consider any $s \in S-\{0\}$; we have

$$
s=a_{1} t(1)+\ldots+a_{m} t(m)
$$


where $a_{j} \epsilon R^{+}-\{0\}$ and $t(j) \epsilon T$ for $1 \leqslant j \leqslant m$. Define a subset $\Sigma$ of $S$ by

$$
\Sigma=\left\{c_{1} t(1)+\ldots+c_{m} t(m): 0 \leqslant c_{j} \leqslant 1 \quad \text { for } \quad 1 \leqslant j \leqslant m\right\}
$$

so that $0 \epsilon \Sigma$ and $\Sigma$ is sequentially compact. From this compactness it is easy to verify the following analog of the lemma proved in Section 2: For any $\eta>0$ there is a $\delta>0$ such that

$$
T_{\sigma}(S(\xi, \delta)) \subseteq S(\xi, \eta) \quad \text { for all } \sigma \epsilon \Sigma \text {, }
$$

where $\xi$ is the equilibrium state.

For each integer $n>0$, let $n(j)$ be the largest integer not greater than $n a_{j}$, so that $n \rightarrow \infty$ implies $n(j) \rightarrow \infty$ for $1 \leqslant j \leqslant m$. Then

$$
T_{s}^{n}=T_{\sigma(n)} T_{t(1)}^{n(1)} \ldots T_{t(m)}^{n(m)}
$$

where $\sigma(n) \epsilon \Sigma$.

Choose neighborhoods $U_{j}$ of $\xi$ such that $T_{t(j)}^{n}\left(U_{j}\right) \rightarrow\{\xi\}, T_{t(j)}\left(U_{j}\right) \subseteq U_{j}$, and $U_{j} \subset S(\xi, \delta)$. Then the proof of Theorem 3 is readily modified to show, for any $\eta>0$, that for any $x \in X$,

$$
T_{s}^{n}(x) \epsilon T_{\sigma(n)}(S(\xi, \delta)) \subseteq S(\xi, \eta)
$$

for all large enough $n$, and that for $U=\stackrel{m}{\bigcap} U_{j}$,

$$
T_{s}^{n}(U) \subseteq T_{\sigma(n)}(S(\xi, \delta)) \subseteq S(\xi, \eta)
$$

for all large enough $n$. This and an application of Theorem B complete the proof.

There is no exploration of analogous extensions of Theorem 2, since the author has not found an intuitively satisfying interpretation of the concept $s \rightarrow \infty$ so essential in stability considerations.

\section{References}

[1] Meyers, P. R., A Converse to the Banach Contraction Theorem, Nat. Bur. Stand. (U.S.), 71B (Math. and Math. Phys.), Nos. 1 \& 2, 73-76 (Apr.-Sept. 1967).

[2] Goldman, A. J., and Meyers, P. R., Simultaneous Contractification, Nat. Bur. Stand. (U.S.), 73B (Math. Sci.), No. 4, 301-305 (Oct.-Dec. 1969).

[3] Meyers, P. R., Contractifiable Semigroups and Lyapanov Functions, in preparation.

(Paper 74B2-323) 